\title{
BEAM LIFETIME OF THE POHANG LIGHT SOURCE
}

\author{
M. G. Kim, C. D. Park, E. S. Park, and T.-Y. Lee* \\ Pohang Accelerator Laboratory, San 31, Hyoja-dong, Pohang, Kyungbuk, 790-784 KOREA
}

\begin{abstract}
Like other third generation light sources, main contributions to the be am lifetime of the Pohang Light Source come from the beam-gas scattering and the Touschek effect. The beam-gas scattering lifetime is measured with extremely low stored current in the long train of bunches. By investigating the vertical gap dependence of the beam-gas scattering lifetime, it $\mathrm{i}$ possible to decompose the beam-gas scattering lifetime to the elastic and inelastic parts. On the other hand, the Touschek lifetime is analyzed using the graph of the measured beam lifetime as a function of time. It i s used that the rate of change of the pure Touschek lifetime is exactly 1 . The deviation from 1 is caused by the beam-gas scattering effect and the radiative electron beam polarization. By analyzing the graph, it is possible to estimate the beam-gas scattering lifetime and the polarization contribution to the beam lifetime.
\end{abstract}

\section{INTRODUCTION}

Beam lifetime is a sensitive function of the beam energy. Pohang Light Source (PLS) is capable of $2-2.5$ $\mathrm{GeV}$ operation. Beam lifetime is a particularly important parameter for a third generation light source of this medium energy range, because beam lifetime may not be long enough to satisfy many users. For higher energy third generation light sources such as Spring8 or APS, beam lifetime is big enough not to cause any inconvenience for users. Therefore the measurement and analysis of beam lifetime is particularly important for PLS. Results of the measurements and analysis of beam lifetime will indicate the proper way of machine operation with respect to beam lifetime and the proper way of increasing beam lifetime without deteriorating other beam parameters. The PLS beam lifetime is governed by the Touschek effect and the beam-gas scattering as in

$$
\frac{1}{\tau}=\frac{1}{\tau_{T}}+\frac{1}{\tau_{V}}
$$

where $\tau_{T}$ denotes the Touschek lifetime and $\tau_{V}$ denotes the beam-gas scattering lifetime. The PLS beam lifetime is apparently dominated by the Touschek effect, like other third generation light sources of comparable energies. But it does not mean that the beam-gas scattering lifetime is not important. The elastic beam-gas scattering lifetime i dependent on the aperture of the beam motion. The decreasing aperture also decreases the elastic beam-gas scattering lifetime. Since an in -vacuum undulator is scheduled to be installed in PLS, the beam-gas scattering lifetime will be more important. In this paper, the beam gas scattering lifetime and the Touschek lifet ime of PLS will be measured and analysed separately. Althogh PLS now operates in two energy modes, 2.0 and $2.5 \mathrm{GeV}$, thi paper will concentrate on the $2.0 \mathrm{GeV}$ operation.

\section{BEAM-GAS SCATTERING LIFETIME}

The beam-gas scattering lifetime $\tau_{V}$ is further decomposed as follows

$$
\frac{1}{\tau_{V}}=\frac{1}{\tau_{C}}+\frac{1}{\tau_{B}},
$$

where $\tau_{C}$ denotes the elastic Coulomb lifetime and $\tau_{B}$ denotes the inelastic bremsstrahlung lifetime. While the bremsstrahlung lifetime depends on the energy acceptance $\Delta E_{r f} / E$, the Coulomb lifetime depends on the aperture (either the physical aperture or the dynamic aperture, whichever is smaller) as well as the lattice parameters, $E$ and $\beta$. Although the energy acceptance $\Delta E_{r f} / E$ itself depends on various lattice parameters and RF parameters, its values are usually a few percent. Hence the bremsstrahlung lifetime is insensitive to storage ring parameters. At a fixed vacuum pressure, it is almost constant. On the other hand, the Coulomb lifetime is very sensitive to the beam energy, aperture size, and the beta function values. Especially the aperture dependence of the Coulomb lifetime is very important for the lifetime analysis. Therefore we denote the Coulomb lifetime as

$$
\frac{1}{\tau_{C}}=\frac{P}{\alpha d^{2}},
$$

where $P$ is the vacuum pressure, $d$ is the acceptance determining half aperture, and $\alpha$ includes all the other parameters. In most cases, $d$ is the vertical half-height of the physical aperture. To measure the beam-gas scattering lifetime, we used extremely low electron beam density to eliminate the Touschek lifetime in Eq. (1). Then we used two vertical beam scrapers (up and down) to investigate the $d$ dependence of the Coulomb lifetime. Using Eq. (2) and (3) in Eq. (1), the beam lifetime $\tau$ can be expressed as

$$
\frac{1}{\tau}=\frac{P}{\alpha d^{2}}+\frac{1}{\tau_{B}},
$$

which is converted to give

$$
\tau=\frac{\alpha \tau_{B} d^{2}}{P \tau_{B}+\alpha d^{2}}
$$

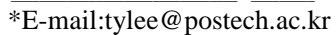


Treating $d$ as a small variable, the above equation $\mathrm{i}$ expanded as [1]

$$
\tau=\frac{\alpha}{P} d^{2}-\frac{\alpha^{2}}{P^{2} \tau_{B}} d^{4}+\mathrm{L} .
$$

The first term is the Coulomb lifetime and the bremmstrahlung lifetime enters into the first order correction term.

If the measured data are fitted to the above formula, it is possible to obtain not only the Coulomb lifetime but also the bremsstrahlung lifetime. The PLS data is compared with this formula in Fig. 1. Figure 1 gives the beam lifetimes measured as a function of $d$ (scraper position from the center) at two different pressures 0.5 nTorr [Fig. $1,(\bullet)]$ and 1 nTorr [Fig. 1, (ם)]. An extremely low electron density $(0.014 \mathrm{~mA} / \mathrm{bunch})$ was used to effectively eliminate the Touschek effect.

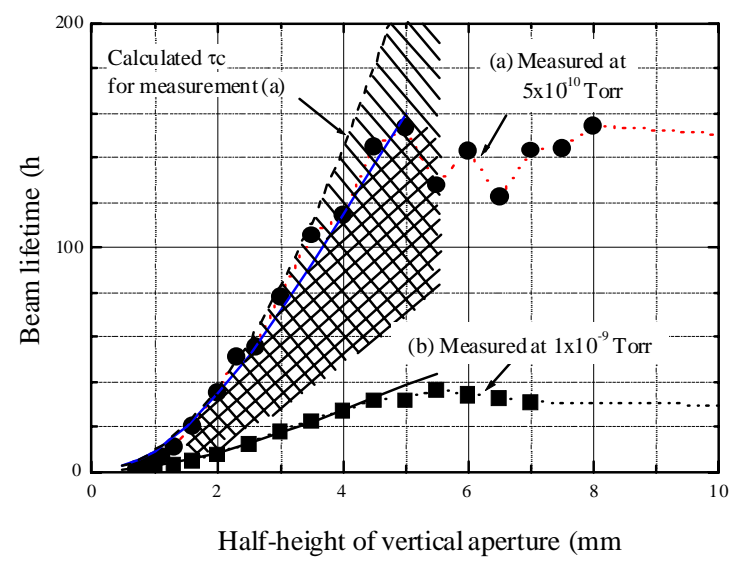

Figure 1: Beam lifetimes measured with vertical beam scrapers at (a) 0.5 and (b) 1 nTorr respectively. The solid line results from data fitting. The dashed line denotes the Coulomb lifetime deduced from the measured data.

If the measured data is to be fitted well to $\tau \approx \alpha d^{2} / P$ at very small $d$ (around 1-2 mm), the pressure $P$ should be elevated a little from the measured values, 0.5 (1.0) nTorr, to 0.7 (2.1) nTorr. The cause of this discrepancy between the measured pressures and the fitted pressures is not clear yet. One possible explanation is the non-uniform distribution of the pressure around the ring. Extrapolating the data fitting results at the very small aperture values to bigger aperture values, we separate the Coulomb lifetimes (dashed line) out of the total beam lifetimes. And once the pressure is chosen, it $\mathrm{i}$ possible to read $\tau_{B}$ from the first order correction. The estimated $\tau_{\mathrm{B}}$ is given by $\tau_{B} \cdot P \approx 410 \mathrm{nTorr} \cdot \mathrm{h}$.

Another important observation in Fig. 1 is that the measured lifetimes do not increase anymore for $d \geq 5$ $\mathrm{mm}$. This strongly indicates the presence of a limiting aperture at $d \sim 5 \mathrm{~mm}$, instead of the $6 \mathrm{~mm}$ undulator halfgap. There are two possibilities. The first possibility is the presence of the vertical dynamic aperture at around $d \sim 5$ $\mathrm{mm}$. But the problem is that this value is a bit smaller than the particle tracking result. The second possibility is that the acceptance-limiting half aperture is still the $6 \mathrm{~mm}$ undulator half-gap, but the beam orbit has some offset $(<1 \mathrm{~mm})$ from the center. Anyway, the effective acceptance-limiting half aperture is now $5 \mathrm{~mm}$. If the fitted pressure $7 \times 10^{-10}$ Torr of Fig. 1 is used instead of th measured pressure $5 \times 10^{-10}$ Torr, we obtain the relation $\tau_{V} \cdot P \approx 115 \mathrm{nTorr} \cdot \mathrm{h}$ for $d=5 \mathrm{~mm}$.

Since the vacuum pressure of the normal operation is from $\sim 0.5$ to $\sim 1.0$ nTorr depending upon the amount of the stored current, all the possible Coulomb lifetime s of PLS are contained in the hatched area of Fig. 1 enclosed by the two dashed lines obtained by data fitting only at small aperture values while all the possible beam-gas scattering lifetimes are contained in the cross -hatched area. In most cases, the beam-gas scattering lifetime $\mathrm{i}$ longer than the Touschek lifetime. As $d$ decreases, the Coulomb scattering effect increases, and for an aperture value of $\sim 1.5-2.5 \mathrm{~mm}$ the beam-gas scattering (Coulomb) lifetime is comparable to or even smaller than the Touschek lifetime.

\section{TOUSCHEK LIFETIME}

The Touschek effect is a loss mechanism caused by the transfer of transverse momentum to longitudinal direction in the scattering of two electrons in a bunch. Therefore the Touschek loss rate is proportional to $N^{2}$ and we may write it as

$$
\frac{d N}{d t}=-a N^{2},
$$

where $a$ is a parameter determined by the $\mathrm{M}$ öller scattering cross section as well as the beam and machine parameters. The Touschek lifetime $\tau_{T}$ is given by

$$
\frac{1}{\tau_{T}}=-\frac{1}{N} \frac{d N}{d t}=a N
$$

The parameter $a$ depends on specific beam models Although the computation of $a$ needs precise knowledge of beam and rf parameters, the resulting Touschek lifetime is not very accurate when compared with measured data. But an exact relation about the Touschek lifetime can be obtained from its rate of change, [1]

$$
\frac{d \tau_{T}}{d t}=\frac{d \tau_{T}}{d N} \frac{d N}{d t}=\left(-\frac{1}{a N^{2}}\right)\left(-a N^{2}\right)=1 .
$$

This relation is independent of $a$ and exact. Hence the Touschek lifetime $\tau_{T}$ is integrated to

$$
\tau_{T}=t+\tau_{T 0},
$$

where $\tau_{T 0}$ is the initial Touschek lifetime. The Touschek lifetime has the universal property that it increase linearly with time, regardless of various beam and machine parameters that determine merely the initial 
value $\tau_{T 0}$. Therefore the dominance of the Touschek lifetime in a given beam lifetime is manifested by ho close the slope is to 1 in the $\tau$ versus $t$ graph.

Using Eq. (6) in Eq. (1) and using the condition that the beam-gas scattering lifetime is much longer than the Touschek lifetime, $\tau$ can be approximated to [2]

$$
\tau ; t\left(1-\frac{t}{\tau_{V}}\right)+\tau_{0}\left(1-\frac{2 t}{\tau_{V}}\right),
$$

where $\tau_{0}$ is the initial beam lifetime given by [2]

$$
\tau_{0}=\frac{\tau_{T 0} \tau_{V}}{\tau_{T 0}+\tau_{V}}
$$

Equation (7) shows that the slope of the $\tau$ versus $t$ graph is always less than 1 . However, in the real $\tau$ versus $t$ of PLS represented by the solid line in Fig. 2, the slope is bigger than 1 (represented by the dotted line) in the early ours. This is caused by the contribution of the radiative electron polarization to the Touschek lifetime. The electron polarization increases the Touschek lifetime. The inclusion of the polarization effect to the Touschek lifetime is given by simply replacing $a$ of Eq. (5) by

$$
a_{p}=a-P^{2} b,
$$

where $P$ denotes the degree of polar ization and the parameter $b$ is determined only by the polarization dependence of the Möller scattering cross section.

The electron polarization can be suppressed by turning on skew quadrupoles which are strong depolarising device. The dashed line of Fig. 2 displays the same PLS beam lifetime with a little bit of skew quadrupole currents. Hence the dashed line represents the PLS beam lifetime with the electron polarization suppressed. Hence the hatched area corresponds to the contribution of the electron polarization to the beam lifetime, which is around $10 \%$ of the beam lifetime. The dotted line represents the pure Touschek lifetime which has slope 1. With the polarization suppressed, the PLS beam lifetime is well fitted to Eq. (7). With this fitting, it is possible to deduce even the beam-gas scattering lifetime as $\tau_{V}: 310 \mathrm{~h}$. Combining this value with the vacuum pressure of the normal operation, we see that the beam gas scattering lifetime obtained this way is reasonably consistent with the value obtained in the previous section, $\tau_{V} \cdot P \approx 115 \mathrm{nTorr} \cdot \mathrm{h}$.

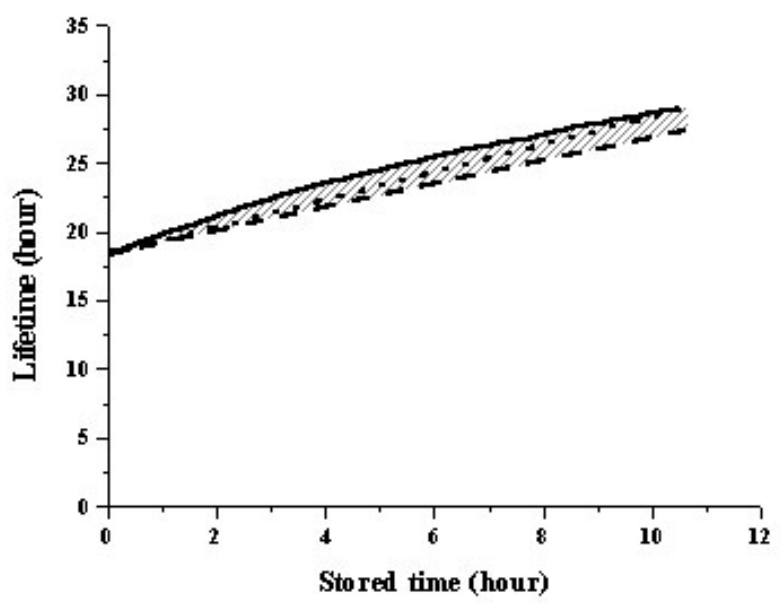

Figure 2: The PLS beam lifetime as a function of time. The solid line denotes the measured beam lifetime, the dotted line denotes the pure Touschek lifetime, and the dashed line denotes the beam lifetime with ske quadrupoles on. The hatched area denotes the polarization contribution to the beeam lifetime.

\section{CONCLUSION}

The PLS beam lifetime has been analysed. The dominance of the Touschek lifetime is now clear from the fact that the slope in the $\tau$ versus $t$ graph is very close to 1.

Using extremely low electron density, it was possible to measure the beam-gas scattering lifetime directly. Further, using two vertical beam scrapers, it was possible to decompose the beam-gas scattering lifetime to the elastic Coulomb lifetime and the inelastic bremsstrahlung lifetime. We obtained $\tau_{V} \cdot P \approx 115 \mathrm{nTorr} \cdot \mathrm{h}$ with the vertical half aperture $d$ of $5 \mathrm{~mm}$ and $\tau_{B} \cdot P \approx 410$ $\mathrm{nTorr} \cdot \mathrm{h}$. Even though the beam -gas scattering lifetime is normally much longer than the Touschek lifetime, the aperture dependence of the Coulomb lifetime makes the beam-gas scattering lifetime still an important factor, especially for the operation of in-vacuum mini undulators.

Analysing the $\tau$ versus $t$ graph, it was possible to obtain the beam-gas scattering lifetime again.

\section{REFERENCES}

[1] C. D. Park, T.-Y. Lee, I. H. Bae, and S. M. Chung, J. Vac. Sci. Technol. A 18, 2722 (2000).

[2] T.-Y. Lee, M. G. Kim, and E. S. Park, to be submitted. 